\title{
Homing in on Axions?
}

\section{A search for dark matter axions with unprecedented sensitivity tests a previously inaccessible parameter range for these hypothetical particles.}

\section{by Frank T. Avignone III*}

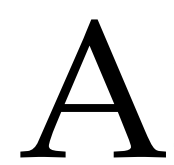

stronomer Fritz Zwicky first proposed dark matter in 1933 to explain the discrepancy between observed rotational velocities of galaxies in the Coma Cluster and expected rotational velocities based on the gravitational potential from the visible matter. Ever since, many studies have built a strong case for the existence of dark matter. Observations of the rotation curves of spiral galaxies, measurements of the temperature fluctuations in the cosmic background radiation, and gravitational-lensing images of distant galaxies clearly show that there is far more mass distributed uniformly throughout galaxies than what can be accounted for by the matter contained in the stars and dust. The question is not whether cold dark matter exists. The question is whether it consists of some type of particle and how such a particle might be detected and identified. The Axion Dark Matter eXperiment $(A D M X)$ has now reported results from its latest search for one of the leading dark matter particle candidates-the axion [1]. Thanks to unprecedented detection sensitivity, ADMX has been able to probe, for the first time, the parameter space favored by both of the two most popular axion dark matter models. While the ADMX Collaboration has found no evidence for axions, the results represent a technical breakthrough in the ability to probe axion parameter ranges that were previously out of reach.

Today, the majority of dark matter searches target a candidate dark matter particle known as the weakly interacting massive particle (WIMP), which is thought to have a mass of around 1-100 $\mathrm{GeV} / c^{2}$, or more-potentially many times larger than that of the proton [2]. WIMPs might be detected because the motion of the Solar System through the galactic halo of dark matter creates an effective WIMP "wind." On Earth, WIMPs may collide with atomic nuclei. In a detector, this collision could generate a potentially measurable signal. Motivated by this possibility, researchers have built dozens of detectors around the world-scintillation detectors, germanium detectors, various types of bolometers, and noble-liquid time-projection chambers. Despite such efforts,

\footnotetext{
*Department of Physics and Astronomy, University of South Carolina, Columbia, SC 29208, USA
}

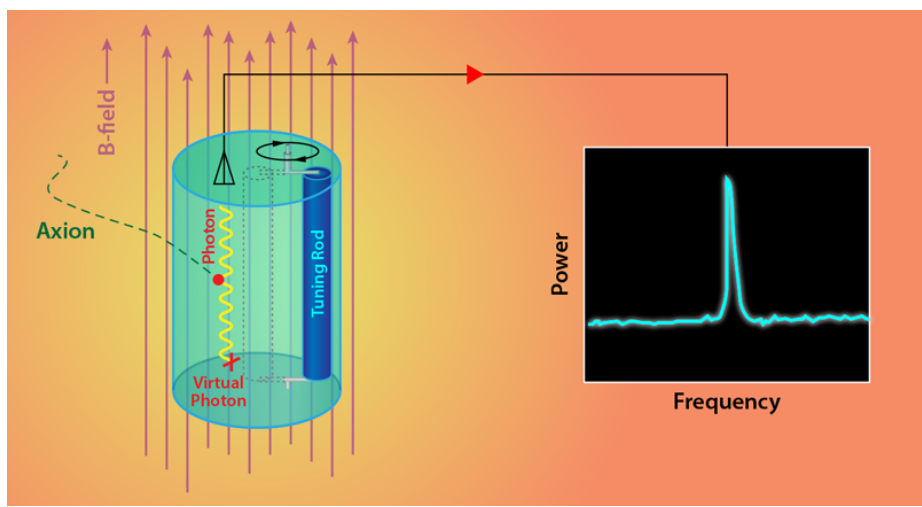

Figure 1: Scheme of the dark matter haloscope used in the ADMX experiment [1]. To search for axions, the resonant frequency of a cavity (left) is shifted by moving rods placed inside it. If the cavity's resonant frequency (right) matched the frequency of photons coupled to axions, the rate of axion-to-photon conversion would be significantly enhanced, generating power in the cavity. (C. Boutan/Pacific Northwest National Laboratory; adapted by APS/Alan Stonebraker)

WIMPs haven't been observed yet. What's more, the Large Hadron Collider in Switzerland has also joined the hunt for WIMPs by looking for WIMP creation in particle collisions and by searching for evidence of supersymmetry-a theory that naturally leads to WIMPs. Such searches have so far come up empty handed. Together, all these results are casting some doubts on the WIMP hypothesis.

An alternative hypothesis is that of the axion. The axion was not initially conceived as a dark matter particle but emerged from a theory that was proposed to solve the strong charge-parity $(C P)$ puzzle. This long-standing problem asks why strong interactions appear to not violate $C P$ symmetry, although such a violation is, in principle, possible. A $C P$ violation in strong interactions would lead to, among other things, an electric dipole moment of the neutron that is $\sim 10^{10}$ times larger than the current experimental bound. In 1977, theorists Roberto Peccei and Helen Quinn [3] proposed a possible solution to the puzzle by introducing a symmetry-breaking mechanism that leads to a new particle, a Goldstone boson called the axion.

Immediately after the Peccei-Quinn article, Steven Weinberg and Frank Wilczek independently worked out the 
physical properties of the axion in the standard model. However, experiments soon ruled out the existence of standard model axions. Later, two models involving "invisible" axions emerged, in which axions interact much more weakly with photons and matter. The two models, known as the Kim-Shifman-Vainshtein-Zakharov (KSVZ) model [4] and the Dine-Fischler-Srednicki-Zhitnitsky (DFSZ) model [5], differ in the strength of the coupling of axions to photons, with the KSVZ coupling $\sim 2.7$ times larger than the DFSZ coupling. Since most axion searches are trying to detect axions through their interactions with photons, DFSZ axions will be more difficult to detect than KSVZ axions.

Shortly after the development of these models, the axion became a leading dark matter candidate. Cosmological models predicted that dark matter axions, produced in the early Universe, would probably have a mass in a range of about $1-25 \mu \mathrm{eV} / c^{2}$-over $10^{15}$ times smaller than the WIMP's. With such a small mass, we would expect tens of trillions of axions per cubic centimeter in our Solar System to account for the observed dark matter density. However, the interactions of axions with ordinary matter and photons are expected to be so feeble that their detection would require extremely sensitive techniques.

The ADMX project, located at the University of Washington in Seattle, is based on a detection scheme introduced in 1983 called the axion "haloscope" [6]. The experiment relies on the conversion of axions into photons in a resonant cavity in the presence of an applied magnetic field (Fig. 1). If this conversion can occur, then its rate will be significantly enhanced when the cavity's resonant frequency matches the produced photon's frequency, which corresponds to the mass-energy of the axion. The production of additional photons in the cavity would lead to the generation of power in excess of thermal noise. Since the photon frequency depends on the unknown axion mass, the experiment continuously tunes the frequency of its resonator by moving rods placed inside the resonator.

Since its inception, the ADMX experiment has gone through several iterations. Until now, however, the detection sensitivity was only adequate to probe axions with the stronger coupling of the KSVZ model. The new results rely on several technical improvements that boosted the experiment's sensitivity. The addition of a dilution refrigerator allowed the team to reduce thermal noise by cooling the cavity to $150 \mathrm{mK}$, while other improvements in the detector's electronics mitigated several sources of systematic uncertainty. With the resulting sevenfold improvement of sensitivity, the collaboration was able to probe a frequency range of $640-685 \mathrm{MHz}$, ruling out the presence of axions with a mass between 2.66 and $2.81 \mu \mathrm{eV} / c^{2}$ and with a range of axion-photon couplings that includes the values assumed by both the DFSZ model and the KSVZ model. This result makes ADMX the first experiment ever to probe and exclude axions with DFSZ couplings in the mass range favored by cosmological models involving cold dark matter.

While ADMX found no evidence for axions, the results bode well for future experimental runs. The team expects to further boost the experimental sensitivity-and thereby improve both the signal-to-noise ratio and measurement speed-by cooling the machine to lower temperatures and by applying a stronger magnetic field. These developments will allow the researchers to probe both DFSZ and KSVZ axions in a much larger mass range than the narrow one reported in this work. The experimental progress reported by ADMX may usher in a new era in axion searches, which could potentially deliver a solution to both the dark matter puzzle and the $C P$ problem of the strong interaction.

This research is published in Physical Review Letters.

\section{REFERENCES}

[1] N. Du et al. (ADMX Collaboration), "Search for Invisible Axion Dark Matter with the Axion Dark Matter Experiment," Phys. Rev. Lett. 120, 151301 (2018).

[2] G. Bertone, D. Hooper, and J. Silk, "Particle Dark Matter: Evidence, Candidates and Constraints," Phys. Rep. 405, 279 (2005).

[3] R. D. Peccei and H. R. Quinn, " $C P$ Conservation in the Presence of Pseudoparticles," Phys. Rev. Lett. 38, 1440 (1977).

[4] J. E. Kim, "Weak-Interaction Singlet and Strong CP Invariance," Phys. Rev. Lett. 43, 103 (1979); M. A. Shifman, A. I. Vainshtein, and V. I. Zakharov, "Can Confinement Ensure Natural CP Invariance of Strong Interactions?" Nucl. Phys. B 166, 493 (1980).

[5] M. Dine, W. Fischler, and M. Srednicki, "A Simple Solution to the Strong CP Problem with a Harmless Axion," Phys. Lett. B 104, 199 (1981); A. Zhitnitsky, "On Possible Suppression of the Axion Hadron Interactions," Yad. Fiz. 31, 497 (1980), [Sov. J. Nucl. Phys. 31, 260 (1980)].

[6] P. Sikivie, "Experimental Tests of the "Invisible" Axion," Phys. Rev. Lett. 51, 1415 (1983).

10.1103/Physics.11.34 